\title{
EL DERECHO HUMANO A LA RESISTENCIA LINGÜÍSTICA
}

\author{
THE HUMAN RIGHT TO LINGUISTIC RESISTANCE
}

\section{MANA SIMIKUNA WAÑUNANPAQ RUNAKUNAP DIRICHUN(1)}

\author{
Alicia Moncada Acosta(2) \\ Universidad Central de Venezuela \\ Fundación para la Justicia y el Estado Democrático de Derecho, México
}

\begin{abstract}
Resumen: Las lenguas han sido históricamente targets (objetivos) de cualquier sistema que quiera imponerse y generar hegemonía cultural. Es por ello que -en el continente americano- desde los inicios del régimen colonial, las lenguas indígenas fueron objeto de persecución y supresión, situación que no se mitigó con la constitución de los estados nacionales. Precisamente porque las lenguas son rocas fundacionales de la etnicidad, ha sido primordial para los pueblos indígenas protegerlas de las arremetidas de proyectos asimilacionistas y etnocidas, siendo la reivindicación de los derechos lingüísticos una herramienta provechosa para tal fin. La intención de este trabajo es presentar una revisión de los estándares internacionales construidos en materia de protección cultural y lingüística, en aras de exponer la utilidad del discurso y estándares de derechos humanos para la resistencia y supervivencia lingüística de los pueblos indígenas.
\end{abstract}

Palabras claves: Pueblos indígenas, derechos lingüísticos, resistencia, derechos humanos.

Abstract: Languages have historically been targets of any system that wants to impose itself and generate cultural hegemony. That is why -in the American continent- since the beginning of the colonial regime, indigenous languages were the object of persecution and suppression, a situation that was not mitigated with

(1) Traduccion: Martin Castillo Collad. Lengua Quechua / Cusco Collao

(2) De origen Wayuu, es investigadora y activista de Derechos Humanos. Fue profesora de la Universidad Central de Venezuela e investigadora en Derechos Económicos Sociales, Culturales y Ambientales de Amnistía Internacional. Actualmente es investigadora de la Fundación para la Justicia y el Estado Democrático de Derecho con sede en la Ciudad de México. También fue integrante de diversas organizaciones de mujeres indígenas de la amazonía y la guajira colombo-venezolana. E-mail: aliciamoncada@gmail.com 
the constitution of national states. Precisely because languages are the foundation rocks of ethnicity, it has been essential for indigenous peoples to protect them from the onslaught of assimilationist and ethnocidal projects, being the protection of linguistic rights a useful tool for this purpose. The intention of this work is to present a review of the international standards built on cultural and linguistic protection, in order to expose the usefulness of the discourse and human rights protocols for the linguistic resistance and survival of indigenous peoples.

Keywords: indigenous peoples, linguistic rights, resistance, human rights.

Pisiyasqa yuyay: Kay qillqan riqsichichkan imaynas tiqsi muyupi kawsaytapas, simikunatapas amachaykuna istandaris nisqamanta, chayqa ima raykun allin kanman rimaykuna, qimiriykunan chay runa dirichus qaqalla sayarinapaq, ñawpa llaqtakunap siminkunapas kawsanallanpaqpuni. Ñawpa simikunaqa, una-unaymantapachan karqanku, munaqniyuqkunap qawasqan chaywan ima munasqankuta wakinkunaman ñitiykunankupaq, chaynata kawsaynillanku mirananpaq.Chayraykun kay -América chiqanpi- colonia qallarisqanmantapacha, ñawpa simikunaqa chinkanankupaq qatikachasqa karqanku, chaykunaqa manataqmi chay Estado Nación rikurimuptinpas pisiyanpaschu, chinkanpaschu. Simikunaqa kayninchikpa sapinmi, allin qaqa tiqnin, chayraykupin ñawpa llaqtakunapaq siminkuqa achwan amachasqa karqa chay wañuchikuq, sipikuq ruraykunamanta, chaymi reivindicación de los derechos linguisticos nisqaqa allinpuni.

Yuyaychaq simikuna: ñawpa llaqtakuna, dirichus linguistikus, qaqa sayay, dirichus humanus. 


\section{Introducción}

La palabra como acto creador y extensión del aliento de vida es un fenómeno constitutivo de la humanidad. El lenguaje moldea y determina, construye mundos, por lo tanto, culturas que dependen de sus sistemas de signos y símbolos para la comunicación de quienes las integran. Es el lenguaje el elemento estructurante de la subjetividad y a su vez el canal que nos permite tejer las relaciones que precisamos para sobrevivir. Ineludiblemente, es la herramienta que nos ha convertido en animales sociales y en generadores de un legado histórico.

Su potencia creadora se repite en los relatos fundacionales de diversos pueblos del mundo. Desde lo -el ser supremo Maorí- que dejó la inactividad para pronunciar las primeras palabras que conformarían al universo hasta el dios hebreo que con su sentencia “hágase la luz" inició los siete días de creación. El lenguaje como fuerza lumínica que destruye a las tinieblas es una constante.

Los pueblos indígenas conocen muy bien el poder de la lengua. Es la roca fundacional del orden simbólico y de la cosmovisión cultural que estructura la etnicidad, entendida -de manera simple-como los elementos socioculturales que aglutinan y a la vez distinguen a los grupos humanos. A diferencia de la raza, que se establece como una categoría que justifica la subordinación a partir de la diferencia fenotípica, la etnicidad refiere a una identidad que se reivindica y es aceptada por todos los miembros del grupo quienes reconocen un pasado y un futuro colectivo, un conjunto de parientes ancestrales y la pertenencia a un espacio geográfico en el que han forjado su historia material, espiritual y cultural.

El lenguaje nos permite hacer de la etnicidad una experiencia y un performance social, cimienta las relaciones que dan continuidad al orden simbólico y las cosmovisiones a través de las generaciones. Por tanto, es vital para la reproducción y la incorporación de nuevos elementos que permiten a las identidades étnicas adaptarse y/o sobrevivir ante los cambios impuestos o endógenos.

Asimismo, el lenguaje al ser un dispositivo que contiene la diferencia sexual (Violi, 1991) refuerza normas y fronteras asignadas culturalmente a los individuos por la acción del género modelando masculinidades y feminidades según las necesidades y requerimientos de la cultura dominante. De igual forma lo hacen los Estados nacionales y sus agentes en la conformación de sujetos étnicos útiles para la (re)producción de la hegemonía, siendo el lenguaje un recurso político (Chiodi y Loncón, 1995) y arma de guerra que, al imponerse, brinda a quienes detentan prebendas sociales una serie de posibilidades para el ejercicio del poder, mientras que a los grupos marginados les priva sistemáticamente de éstas.Además de la imposibilidad de acceder al poder y liderar sus destinos, se suma la restricción de dejar un legado histórico, transmitir el pasado y también pensar un "nosotros" como futuro colectivo. 
Si bien la colonización lingüística como programa político no comenzó con el genocidio americano, sí potenció y coadyuvó al ego conquiro (Dussel, 1994) occidental y su misión imperialista. La colonización lingüística fue uno de los primeros pasos para la instauración del régimen colonial, pues muy a pesar del decreto del 3 de julio de Felipe II a favor del bilingüismo y una primera etapa favorable a las lenguas indígenas fomentada por algunos de los misioneros precursores, ya para 1571, el Consejo de Indias autorizaba la reducción de los idiomas indígenas y la bibliocastía de los códices mayas.

Posteriormente, los Estados nacionales continuaron reproduciendo $-\mathrm{y}$ profundizando- la colonización lingüística ya no desde el programa conquistador sino como instauración de una civilidad homogeneizante basada en el paradigma del buen ciudadano burgués, blanco y noreurocéntrico, realidad en la que los pueblos indígenas no tienen, ni quieren, ser parte.

Por ello, reivindicamos la importancia de luchar contra la hegemonía lingüística, recordando que el silenciamiento de los idiomas es también una forma de etnocidio. Precisamente, porque se hace necesario tener herramientas para enfrentar estas manifestaciones del colonialismo, algunos espacios del movimiento indígena pan-indianista se han apropiado del discurso de los derechos humanos, entre los que se encuentran los derechos lingüísticos, así como los estándares internacionales que legitiman su protección.

En este trabajo nuestra intención es vislumbrar la utilidad del discurso de los derechos humanos y los instrumentos normativos para la resistencia y supervivencia lingüística de nuestros pueblos. Haremos una revisión del discurso de los derechos y su impronta occidental, así como analizaremos los estándares internacionales construidos en materia de protección cultural, todo en aras de exponer tanto las potencialidades como las limitaciones en la lucha por la reivindicación de nuestras diferencias culturales.

\section{Los pueblos indígenas y el discurso de los derechos humanos}

Desde el pensamiento indianista y anticolonial podemos dar una larga discusión crítica sobre los derechos humanos. Es posible comenzar con su origen eurocéntrico y que uno de sus pilares fundacionales sea la constitución de los Estados. Coincidiendo así con Walter Mignolo (2003) cuando afirma que en esta narrativa siguen vivas las viejas normas occidentales de civilización, pero ahora reivindicando normas globales de calidad de vida.

Tampoco se puede obviar que la tradición liberal -imperante en el sistema de derechos humanos- privilegia los derechos fundamentales que resguardan los valores occidentales del individualismo y racionalismo, dejando para discusiones 
menores los derechos colectivos que buscan proteger el bien común. Sin entrar en mucho detalle, ontológicamente estamos frente a la disputa entre el holismo y el individualismo, siendo para el holismo la comunidad lo que antecede a la individualidad y por lo tanto le es superior en importancia. Esta discusión que tiene sus raíces en las diferencias que surgen entre el pensamiento kantiano y hegeliano, así como otras corrientes históricas (romanticismo e ilustración), nos ha obligado a pensar en quien(es) puede(n) ser sujeto(s) de derecho(s). Siendo este un punto vital para los pueblos indígenas, pues -en parte- de allí surgen nuestras reticencias y/o críticas al discurso liberal de los derechos humanos, pues no nos interesa la reivindicación del "ser" como entidad aislada sino como una parte vital del "nosotros".

Para el racionalismo liberal, el sujeto de derechos es un ser abstracto o genérico que se supone igual a todos los demás humanos, esta ficción encubre que los atributos adjudicados a esa abstracción son el resultado de la generalización de valores o rasgos de los grupos hegemónicos, así como la preeminencia de una igualdad jurídica supuestamente imparcial ante las particularidades económicas, culturales, étnicas, política o raciales.

Considerar a los individuos como entidades abstractas, y pretender que la aspiración kantiana de la "ley general de libertad" sea una realidad fáctica, invisibiliza que todos los seres humanos estamos atravesados y dependemos de contextos sociales e históricos, siendo esto importante para los pueblos indígenas ya que determina en gran medida nuestra existencia actual y futura.

La concepción liberal del sujeto de derecho abstracto acarrea un problema para los pueblos indígenas o para cualquier grupo humano que revindique colectivamente diferencias culturales o particularidades que son divergencias de lo considerado mayoritario o "normal". Sin embargo, esto no significa que estemos en contra de que individualidades también reclamen protección, a propósito de la exaltación de ciertas tradiciones y valores aceptados por los actores de poder dentro de grupos considerados minorías, tal es el caso de la infancia, las mujeres e incluso la diversidad sexual en los pueblos indígenas.

Frente a esta discusión, los pueblos indígenas abogan por la construcción de una nueva cultura de Derecho basada en el pluralismo jurídico, que reconoce a los sujetos colectivos. Si bien no es la intención de este trabajo entrar en la discusión sobre la interpretación de los derechos colectivos y su importancia para los pueblos indígenas, nos basta con puntualizar que éstos no son concebidos por la tradición liberal como parte del sistema internacional de los derechos humanos ya que se comprenden como derechos individuales de ejercicio colectivo. 
Esta situación choca con la naturaleza colectiva de los pueblos e identidades étnicas y a la vez nos hace sujetos que desafían las teorías liberales convencionales, cuya idea base es que la protección de un grupo -como entidad cultural y legal diferenciada- no es necesaria mientras se garanticen derechos a los individuos. A propósito, Mattias Ahrén (2010), presidente del consejo Sami, aclara que la teoría liberal ignora por completo las estructuras sociales colectivistas de los pueblos indígenas, un sistema así no es funcional para la protección integral, por ejemplo, ante los proyectos etnocidas y de asimilación cultural que devienen del colonialismo.

Afirma Ahrén que en la visión liberal no existe el derecho a un tratamiento diferenciado, aunque se pertenezca a una alteridad étnica. Para ejemplificar esta idea, indica que la totalidad de las personas en un país tienen el derecho a hablar la lengua nacional, pero cuando no hay un marco normativo favorable para las diferencias culturales, una persona no podría reivindicar hablar su lengua materna si no es la hegemónica.

Entonces, ¿servirán realmente los derechos humanos como una herramienta para la resistencia lingüística de los pueblos indígenas?

Compartimos la postura de Upendra Baxi (2002), los derechos humanos tienen un potencial desestabilizador de concentraciones de poder que producen injusticias y reconocen -a nivel declarativo inicialmente- el sufrimiento de sujetos que han estado históricamente vulnerados por sistemas opresivos cimentados en la discriminación racial, étnica, cultural y/o lingüística. Así es que pueden convertirse y utilizarse como una "práctica insurrecta" como lo define Baxi en la medida en que los sujetos se apropian del discurso y lo instrumentalizan como espacio de transformación política.

Es por ello que, como resultado de una serie de esfuerzos de los pueblos indígenas y aliados del movimiento, se logró generar una evolución -por lo menos en el plano de lo formal- del Derecho internacional hasta considerar que tenemos el derecho de seguir (re)produciendo nuestra vida cultural y espiritual en paralelo a la sociedad hegemónica. Esto se traduce en una serie de estándares para la protección de los pueblos indígenas en el sistema de derechos humanos, constituyéndose los derechos culturales en un pilar fundamental de las reivindicaciones.

Partiendo de que los pueblos indígenas son protagonistas del reconocimiento de los derechos colectivos y su poder para subvertir el discurso liberal imperante, los derechos lingüísticos son parte integral del desmantelamiento del accionar de los poderes imperiales. Veamos pues en qué medida coadyuvan a dicha causa la consecución de tales garantías. 


\section{Derechos lingüísticos: ¿herramientas para la resistencia?}

Aunque en la Declaración Universal de los Derechos Humanos (1948) no se indica el derecho específico al uso del idioma, sí se establece la prohibición de la discriminación por razones lingüísticas (artículo 2). En la misma tónica encontramos que la Declaración de la Organización de Estados Americanos sobre los Derechos y Deberes del Hombre (1948), el Pacto Internacional de Derechos Civiles y Políticos de Naciones Unidas (1966), en sus principios de no discriminación, determinan que las lenguas no pueden ser razones para limitar derechos a los individuos.

Encontramos especificado el derecho al idioma como parte de los artículos que salvaguardan la no discriminación en la Convención Americana sobre los Derechos Humanos (1969) y el Protocolo Adicional de la Convención Americana sobre los Derechos Humanos en materia de Derechos Económicos, Sociales y Culturales (1978), aunque este último no haga ningún tipo de alusión a la particular situación lingüística de los pueblos amerindios.

Tanto la Declaración de las Naciones Unidas de las Personas pertenecientes a Minorías (1992), como el Convenio 169 de la Organización Internacional del Trabajo (1989), incluyen acciones positivas en sus artículos. Eso significa que no solo se protege de la no discriminación sino que los Estados deben garantizar condiciones legislativas, institucionales y de otros tipos, para el ejercicio efectivo de esos derechos.

La Declaración de los Derechos Lingüísticos (1996) al ser un instrumento que surge del trabajo conjunto entre la sociedad civil, la academia y la Unesco, pareciera que está construida desde el lenguaje matizado y poco contundente que caracteriza a muchos estándares de protección de los derechos humanos. Empero, se expresa específicamente sobre las tendencias homogeneizadoras de los estados nacionales, la hegemonía lingüística de los poderes imperiales y las consecuencias del poder colonial, considerándose en sus palabras preliminares que:

"La invasión, la colonización y la ocupación, así como otros casos de subordinación política, económica o social, implican a menudo la imposición directa de una lengua ajena o la distorsión de la percepción del valor de las lenguas y la aparición de actitudes lingüísticas jerarquizantes que afectan a la lealtad lingüística de los hablantes; y considerando que, por estos motivos, incluso las lenguas de algunos pueblos que han accedido a la soberanía están inmersas en un proceso de substitución lingüística por una política que favorece la lengua de las antiguas colonias o de los antiguos poderes imperiales" (Versión digital) 
Ya que no hay nada más aterrador para los nacionalismos que la heterogeneidad cultural y lingüística, la Declaración además hace eco de "la secular tendencia unificadora de la mayoría de estados a reducir la diversidad y a favorecer actitudes adversas a la pluralidad cultural y al pluralismo lingüístico" (Preámbulo), lo que significa una denuncia de los programas monoétnicos y monolingües que son la base de los nacionalismos radicales, cuyas políticas vernáculas fomentan la discriminación xenófoba y de los grupos que revindican diferencias culturales.

También es posible interpretar que en la Declaración se hace alusión a la imposición lingüística como una forma de discriminación, que distinguimos ha jugado un papel importantísimo en procesos de persecución, un crimen contra la humanidad establecido por el derecho internacional que genera acciones inhumanas y atenta contra la dignidad de las personas -como individuos o parte de un colectivo- en base a sus preferencias religiosas, etnicidad, sexualidad u opinión política.

La persecución es una forma de violencia estatal que históricamente ha convertido a los idiomas de los grupos declarados enemigos en targets (objetivos) de guerra. Son entonces los estándares internacionales importantes no solo en la exigencia de protección y justicia, sino también porque "dejan al descubierto la diferencia epistemológica colonial y la estructura del conocimiento creada y reproducida en cada creación del sistema-mundo moderno/colonial" (Mignolo, 2003: 373).

Los pueblos indígenas tuvieron que esperar hasta la Declaración de las Naciones Unidas sobre los Derechos de los Pueblos indígenas (2007) para ver el derecho al idioma contemplando aspectos más específicos que los abordados por el Convenio 169 (1994) y el Convenio 107 (1957). Esta Declaración indica bajo el mismo espíritu de los convenios mencionados que:

1. Los pueblos indígenas tienen derecho a revitalizar, utilizar, fomentar y transmitir a las generaciones futuras sus historias, idiomas, tradiciones orales, filosofías, sistemas de escritura y literaturas, y a atribuir nombres a sus comunidades, lugares y personas, así como a mantenerlos.

No obstante, establece la necesidad de que los Estados adopten medidas políticas y prácticas, así como condiciones institucionales, para el ejercicio de este derecho:

2. Los Estados adoptarán medidas eficaces para asegurar la protección de ese derecho y también para asegurar que los pueblos indígenas puedan entender y hacerse entender en las actuaciones políticas, jurídicas y administrativas, proporcionando para ello, cuando sea necesario, servicios de interpretación u otros medios adecuados. 
Vemos entonces dos categorías de derechos lingüísticos, unos orientados a garantizar la no discriminación de los individuos y otros relacionados con la promoción y creación de medidas positivas que aseguran un fortalecimiento lingüístico frente a los idiomas hegemónicos.

En el artículo de la Declaración sobre la educación y los medios de comunicación, el derecho al idioma juega un papel fundamental:

1. Los pueblos indígenas tienen derecho a establecery controlar sus sistemas e instituciones docentes que impartan educación en sus propios idiomas, en consonancia con sus métodos culturales de enseñanza y aprendizaje.

2. Los indígenas, en particular los niños, tienen derecho a todos los niveles y formas de educación del Estado sin discriminación.

3. Los Estados adoptarán medidas eficaces, conjuntamentecon los pueblos indígenas, para que las personas indígenas, en particular los niños, incluidos los que viven fuera de sus comunidades, tengan acceso, cuando sea posible, a la educación en su propia cultura y en su propio idioma.

Los derechos lingüísticos son ejercidos individualmente en la medida que los sujetos reivindican el poder comunicarse en su lengua materna sin que por ello sean discriminados, y en el plano colectivo como defensa de la diferencia lingüística que les caracteriza como grupo.

Lamentablemente, la Declaración no es un instrumento vinculante, sino que forma parte de acuerdos conocidos como softlaw que si bien se enmarcan en estándares de derechos humanos convenidos por la comunidad internacional, su incumplimiento no genera responsabilidad jurídica estatal. Aunque no exista la obligación jurídica, estos acuerdos generan una responsabilidad ética y política sirviendo a quienes exigen justicia y reparación como instrumentos para ejercer presión en el sistema internacional de derechos humanos. Son pues herramientas desestabilizadoras del status quo y tienen un potencial, siguiendo el pensamiento de Baxi, de servir para los movimientos de resistencia frente a la mutación del pensamiento moderno y sus nuevas formas de opresión ante las que se necesita la construcción de nuevos derechos.

\section{Conclusiones: derechos lingüísticos de los pueblos indígenas, sus alcances y desafíos}

Lo dejó bien en claro Rodolfo Stavenhagen (2013): luego de la euforia de la Declaración, la batalla por los derechos indígenas -que no deja de librarse en Naciones Unidas- continúa. A pesar de la multiplicidad de garantías promulgadas, 
las agendas estatales asimilacionistas siguen su curso, ahora matizadas y reacomodadas ante la presión moral que implican los estándares de protección a los pueblos indígenas. Se aúna a esto la agresiva avanzada del extractivismo y la carrera desenfrenada de la expansión capitalista que amenazan la vida física no solo de nuestros pueblos, sino de todos los seres que habitan la tierra.

Ante este panorama, parece casi un lujo hablar de los derechos lingüísticos cuando a diario atestiguamos el asesinato de defensores y defensoras indígenas en la protección de los territorios y los dones de la naturaleza.El escenario nada alentador nos hace pensar que la agenda indianista debe concentrar casi todos sus esfuerzos en salvar los últimos reductos donde la tierra respira en libertad y en los que fungimos como guardianes.

Pero la resistencia trasciende el tema territorial. Mantenernos firmes en la defensa de nuestros idiomas también permite desmantelary subvertir las relaciones de dominación propias del colonialismo y la colonización de la memoria (Mignolo, 1993). Siendo los derechos lingüísticos, como ya indicamos, herramientas útiles en procesos, por ejemplo, de litigio ante las manifestaciones del asimilacionismo estatal de intenciones etnocidas -por acción u omisión-que se expresan más allá del desplazamiento territorial. Los derechos implican una barrera, así sea declarativa, para un grupo hegemónico que trata de acabar "con la posibilidad de transmitir la lengua de una generación a otra mediante la imposición de su propia lengua bajo la apariencia de una lengua común" (Macedo et al, 2005: 40) exterminando también la secuencia cultural y propiciando el desarraigo de las raíces culturales. (Ibídem, 2005)

Es imposible negar que la penetración de las culturas y lenguas hegemónicas en los espacios sociales tradicionales de los pueblos indígenas contribuye al desplazamiento sistemático de estas comunidades lingüísticas hacia la(s) lengua(s) oficial(es), por lo que generalmente, y por acción de los cambios generacionales, terminan suplantando a las lenguas nativas. De esta manera, los integrantes de las comunidades lingüísticas indígenas, conscientes de la importancia de manejar el lenguaje oficial, muchas veces decantan por negarse a seguir reproduciendo los idiomas nativos en base a una supuesta inutilidad para la vida práctica de las siguientes generaciones y sus relaciones con el mundo no indígena. La necesidad de crear interlocutores válidos y eficientes con el sistema mundo y la aldea global impulsa al menoscabo funcional y estructural de las lenguas indígenas, eso implica que ante este estancamiento se opte por introducir cada vez más vocabulario exógeno y elementos que terminan por convertir al idioma en una lengua minoritaria regresiva.

La educación formal, aunque es parte de los espacios que han tributado con eficacia al asimilacionismo de los estados nacionales, representa para muchos indígenas una oportunidad de desarrollar potencialidades propias y comunitarias, 
perdiéndose de vista que lo que parece una decisión personal de aceptar la imposición de una política educativa homogeneizante es en realidad el resultado de una estrategia estatal de desaparición de la diversidad cultural.

Además del asimilacionismo, otro de los escollos contra los que se estrella el ejercicio de los derechos lingüísticos es la idea de que éstos forman parte del patrimonio cultural de las naciones. La fagocitación de las lenguas indígenas en el discurso, generalmente progresista, de algunos Estados nacionales ha provocado que se les folklorice y patrimonialice, en vez de fungir como un aporte clave para la construcción de sistemas políticos plurales, que incluso trasciendan la ya gastada cultura democrática.

Frente a la perspectiva de las lenguas indígenas como parte del patrimonio cultural de las naciones encontramos que los gobiernos pueden usar "conquistas" como la declaración de patrimonio inmaterial como parte de una fachada proindígena, a pesar de que la realidad indique lo contrario. Tal es el caso del idioma Mapoyo, propuesto por el gobierno venezolano y declarado el 2013 patrimonio inmaterial por la Unesco. Paradójicamente la propuesta y declaración se realizó en el marco de la aprobación del Decreto № 2.248 que legaliza la extracción minera en la Amazonía venezolana y que pone en riesgo la vida de indígenas y los ecosistemas que allí hacen vida. El Mapoyo, a pesar del escaso número de hablantes podría sobrevivir a la extinción, fue usado estratégicamente para el encubrimiento de problemas más complejos relacionados con los megaproyectos extractivistas decretados en ese territorio.

En este orden de ideas, creemos que las lenguas no son solo celebrables en su día internacional o legados culturales más útiles a la arqueología que a nuestra vida práctica, son elementos políticos de los que nos asimos cuando nos reivindicamos colectivamente, útiles cuando damos la batalla instrumentalizando el discurso y herramientas de los derechos humanos contra el colonialismo. Las lenguas nos entrelazan en un pasado, presente y futuro compartido que es necesario defender de la misión civilizatoria de occidente incluso en sus propios dominios.

Este es el horizonte ante el que vale esgrimir la caja de herramientas de los derechos humanos para el reconocimiento de la taxativa necesidad de crear políticas interculturales que traten de resolver los desequilibrios lingüísticos. La política idiomática que los derechos protegen parte de hacernos conscientes globalmente de la diversidad étnica, cultural y lingüística que nos conforma, con fines de transitar hacia una convivencia justa que reconoce el valor de las diferencias y sus aportes al buen vivir de todas y todos.

Los alcances de poseer una herramienta como los derechos lingüísticos dependen de la apropiación y uso estratégico que realicen los pueblos indígenas 
del discurso de los derechos humanos, que, como ya vislumbramos, adolecen de profundas falencias, pero, a la vez, contienen un potencial subversivo útil. Que sirvan entonces para alimentar la rebeldía de los pueblos en resistencia.

\section{Referencias}

AHRÉN, M, (2010) "Introducción a las disposiciones sobre tierras, territorios y recursos naturales de la Declaración de la ONU sobre los derechos de los pueblos indígenas" en Charters. C y Stavenhagen R. (2010) El desafío de la declaración. Historia y futuro de la declaración de la ONU sobre Pueblos Indígenas. Copenhague: IWGIA.

BAXI, U, (2002) The future of human rights. Oxford: Oxford University Press.

CHIODI, F. y LONCÓN, E, (1995) Por una nueva política del lenguaje: temas yestrategias del desarrollo lingüístico del mapudugun. Santiago de Chile: Pehuén Editores y Ediciones de la Universidad de la Frontera.

DUSSEL, E, (1994). 1492. El Encubrimiento del Otro. La Paz: Plural Editores.

MACEDO, D, DENDRINOS, B y GOUNARI P, (2005) Lengua, ideología y poder: La hegemonía del inglés. Barcelona: Gráo.

MIGNOLO, W, (2003) Historias locales / diseños globales: Colonialidad, conocimiento subalternos y pensamiento fronterizo. Madrid: Akal.

. (1993) "La colonización del lenguaje y la memoria: complicidades de la letra, el libro y la historia" en Zavala, I.M (Coord) Discursos sobre la "Invención" de América. Ámsterdam: RodopiPublishers.

ORGANIZACIÓN DE LAS NACIONES UNIDAS, (1996) Declaración de los Derechos Lingüisticos. Versión digital.

ORGANIZACIÓN DE LAS NACIONES UNIDAS, (2007) Declaración de las Naciones Unidas sobre los derechos de los Pueblos indígenas. Versión digital.

ORGANIZACIÓN INTERNACIONAL DEL TRABAJO, (1989) Convenio Nº 169 sobre Pueblos Indígenas y Tribales. Versión digital.

STAVENHAGEN, R, (2013) "Pueblos indígenas: retos después de la batalla" en Gómez, F y Berraondo, M. Los derechos indígenas tras la Declaración: El desafío de la implementación. Bilbao: Publicaciones de la Universidad de Deusto.

VIOLI, P, (1991) El infinito singular. Madrid: Cátedra. 